\title{
A Collaborative Road Traffic Regulation Approach Using a Wireless Sensor Network
}

\author{
Nouha Rida, Mohammadia School of Engineers, Morocco* \\ Abderrahim Hasbi, Mohammadia School of Engineers, Morocco
}

\begin{abstract}
In this paper, the authors detail and evaluate a coordinated approach to determining the sequence and duration of green lights at several intersections as part of the intelligent transportation system. The authors present the architecture of a wireless network used to track variations in adjacent intersections. The algorithm exploits the collected data to determine the sequence of the green lights based on three objectives: (1) reduce the length of queues in the intersection, (2) prioritize sending vehicle flows to intersections with lower traffic density than the most congested, (3) synchronize traffic signals between adjacent intersections to create green waves. Traffic simulations have been simulated by the SUMO traffic simulator. They show that the solution manages to react to traffic change and reduce waiting time compared to isolated control strategies.
\end{abstract}

\section{KEYWORDS}

Coordinated Traffic Light Control, Intelligent Transportation System, Smart Mobility Synchronized Traffic Light Control, Traffic Light Control, Wireless Sensor Network for Smart Mobility

\section{INTRODUCTION}

We are interested in the management of traffic lights in an urban road network to reduce congestion and decelerations. Traditionally, traffic signal intersections are managed by controllers who define and implement a predetermined sequence (called a traffic signal plan), alternating green and red lights without taking into account traffic variations.

In some urban areas, detectors count the number of vehicles in each lane of an intersection. These devices enable the dynamic management of traffic light plans, using so-called adaptive methods that react to traffic conditions. Indeed, knowledge of the distribution of the number of vehicles per lane makes it possible to estimate the most appropriate values at a given moment of variables such as the green light time or the sequence of lights allowing to reduce, for example, the average waiting time.

The current architectures of these systems are generally organized around a traffic light controller who can make a local decision or communicate his information to a control center capable of transmitting a policy in return.

This operating mode is that of many operated sensor networks. In this paper, we study how to organize this management while taking into account the interactions between nearby intersections using a wireless sensor network. The sensor network considered here is composed only of fixed sensors, in order to avoid problems related to the critical mass of users to be reached before reaching a functional application. However, it is possible to integrate the vehicles into the architecture in a second step. In the case of Intelligent Transportation Systems (ITS), the use of numerous low-cost sensors represents 
an alternative to electromagnetic loops. The deployment density that can be achieved allows local detection and treatment of congestion problems, resulting in a distributed and more responsive ITS that does not rely on a single decision entity.

The paper is organized as follows: after the introduction, we present the problem and context in the second section. After that, the third section discusses related literature and presents system network. While in the fourth one, we propose a wireless sensor network topology for traffic monitoring that ensures the availability of traffic status for a network of road intersections. Both the fifth and sixth sections present our coordinated traffic light control algorithm to determine the sequence of phases and the time of green light according to the traffic on the intersections network. Finally, in the seventh section, we evaluate the proposed method via the SUMO simulator, and we demonstrate its effectiveness by comparing the results to other approaches.

\section{RELATED WORKS}

As mentioned in the previous section, there are two main types of traffic control management. There is a fixed time controller, with a predetermined and fixed cycle, and there is also an adaptive controller that modifies the cycle sequence and the duration of the phases as a function of the numbers of the vehicles present on each lane.

The authors in (Fayez et al., 2020) propose a tracking system for hidden objects for real-time monitoring. This hybrid system is composed of two techniques: a fast technique, circulating structure kernels with color names and an efficient real-time object tracking (ROT) technique aware of occultation.

In (Yousef et al., 2010), the authors propose an adaptive controller based on the theory of the queue and the number of vehicles in each direction as a decision criterion. However, the controller treated in (Helbing et al., 2005) is based on a dynamic fluid model. The system proposed in (Odeh, 2013) detects the level of congestion and abnormal situations in two main highways and for four intersections, and makes a real-time decision that determines the green-light interval for each traffic light at each intersection based on the genetic algorithm.

A self-organization of traffic lights based on historical traffic status data is presented in (BurguilloRial et al., 2012; Yousef et al., 2019). The controller in (Zhu et al., 2016) is cooperative between a network of intersections and semi adaptive. Hence, the decision was made once per cycle based on the number of vehicles in the intersections and on the roads connecting the intersection with its neighbors. All phases in the system in (Rithesh et al., 2018) have a constant duration of 30s, and the choice of the next phase is done according to a decision tree. In (Collotta et al., 2015; Salman et al., 2018; Zou et al., 2009), the authors propose adaptive controllers based on fuzzy logic.

The method proposed in (Zhou et al., 2010) selects the sequence of phases composing a cycle according to several criteria which are the presence of priority vehicles, the duration of the periods without detection of new arrivals, the causes of famine, the total time of waiting and the length of the queues. In (Rida \& Hasbi, 2018a) and (Rida \& Hasbi, 2018b), we inspired by the "Smallest job first" method of scheduling tasks to run them by a computer processor. We proposed the "Smallest phase first" algorithm which gives priority to phases with the smallest queues. Subsequently, in (Rida et al., 2020b; Rida et al., 2018), we integrated the waiting time in the algorithm proposed in (Rida \& Hasbi, 2018b).

In (Belkeziz \& Jarir, 2020), the authors present a literature review of existing approaches to IoT coordination.

In (Belbachir et al., 2019; Jin \& Ma, 2018; Jin \& Ma, 2019; Li, Shahidehpour, Bahramirad et al, 2016; Liu et al., 2017; Tan et al., 2018), the authors find that the traffic road control problem at the intersections is a multi-agent system, in which each agent adjusts the traffic lights according to the traffic variations in real time. 
For intersections control, the main difference between the proposed multi-agent systems models in the literature exists in their agent design approaches. They choose to classify different entities as agents, such as an intersection, the traffic light controller, or a vehicle.

In order to adapt traffic lights to traffic changes, researchers use reinforcement learning (RL) methods (Aslani et al., 2018; Gao et al., 2016; Ge et al., 2019; Ha-li \& Ke, 2017; Li, Lv, \& Wang, 2016; Liang et al., 2019; Mousavi et al., 2017; Van der Pol \& Oliehoek, 2016; Zhou et al., 2019). Traditional reinforcement learning is difficult to use and implement due to two main challenges, the first is the presentation models of the environment, the second is the modeling of the link between, and the third is the decision and the environment. In order to meet these challenges, researchers use deep reinforcement learning techniques, such as Q-Learning and Deep Q-Learning (Li, Lv, \& Wang, 2016; Van der Pol \& Oliehoek, 2016; Wei et al., 2018).

Problems using reinforcement learning techniques are modeled in most cases as a Markov decision process which chooses its decision according to three factors that are the system state (S), the action (A), and the (R) reward function. In the literature, there are several works based on RL, which propose strategies, that provide state-to-action mapping to choose the most cost-effective action for a given state. The goal of those works is to find the optimal action with the greatest overall reward. These approaches are generally distinguished by the choice of the three factors listed below. For example, for the state, there are those who choose the number of vehicles in the queues, or the waiting time, etc. Using various reward functions can be mono-objective or multi-objective.

Neural networks are inspired by the functioning of biological neurons, and implement learning by experience. In the case of road traffic, several authors have studied this scheme (examples: (Spall \& Chin, 1997)). Here, it is a question of a quick classification, and learning to improve them, rather than going through a traditional modeling.

In another study (Rida et al., 2020a), the authors proposed a multi-objectives scheme ant colony optimization algorithm for traffic light (ASTL) to control dynamically the cycles of traffic lights. This solution controls the traffic light by using nature-inspired solution called ant colony optimization.

In (Rithesh et al., 2018), the authors present two methods. While the first one detects the vehicles by a processing of the video captured using cameras, the other one takes the decision of the next green light with a constant duration of 30s. The decision is based on a decision tree according to the number of vehicle and the famine (the selection number per unit of time). The traffic control method proposed in (Rithesh et al., 2018) focuses only on the choice of the next phase with a fixed green time for all phases. It proposes a single objective optimization based on a single criterion, which is the number of vehicles in each lane.

In (Garg \& Kaushal, 2017), the authors propose a real-time traffic monitoring system that combines a wireless sensor network with several fuzzy logic controllers. For each phase, the authors use a fuzzy logic controller to determine the duration of its green light.

An intelligent traffic control system based on an intelligent intersection is developed in (Barzilay et al., 2018). The system reacts and determines the sequence of green lights according to the number of vehicles in each direction, the types of these vehicles (e. g. emergency vehicles), and the social characteristics of the passengers (e. g. late students).

To solve the problem of traffic light synchronization, in (Gao et al., 2016; Kumaran et al., 2019), the authors use a centralized approach. The main objective of the optimization method, proposed in the first work, is to minimize the total time delay of the network in a given finite horizon, while in the second one, is to optimize the vehicle flow in a fixed lapse of time. Similarly, the signaling control method proposed in (Younes et al., 2016) aims at minimizing queue lengths and maximizing throughout. In (Shi et al., 2016), Relative backlog rate-based signaling has been proposed. The algorithm proposed in (Yen et al., 2018) is based on the delay to optimize the flow of vehicles crossing the intersection during the green light. In (Ahmad et al., 2016), the authors proposed two methods, the Minimum Destination Distance First (MDDF) and the First Minimum Average Destination Distance (MADDF) to maximize the flow of traffic. 
In (Younes \& Boukerche, 2018), the authors propose a traffic light management algorithm according to the real-time distribution of vehicle flows around the intersection. The propose algorithm also allows the management of the emergency vehicle passage by defining the sequence of phases in such a lane as to allow the emergency vehicles approaching the intersection to go green without stopping.

In (Faye et al., 2012a), the authors are interested in a network covering several intersections, and they extend their propose solution in (Faye et al., 2012b) to fit an intersection network. They proposed a distributed algorithm that allows sensors to constantly cooperate and adapt the traffic lights strategy to traffic conditions. In order to simplify and make their algorithm effective, they define a score of three criteria to be used at several intersections in order to evaluate each movement of an intersection and to select the most important ones according to the vehicles presented in the all intersections. The traffic information used in these controllers is collected by inductive loops (Zhu et al., 2012), cameras (Odeh, 2013; Rithesh et al., 2018), radars, VANET (ad hoc network for vehicles) (Jing et al., 2017; Khanafer et al., 2009) or a wireless sensors network (Faye et al., 2012a; Yousef et al., 2010; Zhou et al., 2010).

For a controller based on a wireless sensor network, the topologies proposed in the literature in (Yousef et al., 2010; Zou et al., 2009) are composed of a sensor or two per direction to specify the number of vehicles in the queue. In (Tubaishat et al., 2008), the authors studied the influence of the choice of a topology with a sensor per direction and two sensors per direction on the average waiting time. They found that a topology with two sensors gives a reduced vehicle waiting time compared to that with a sensor per direction.

\section{PROBLEM AND CONTEXT}

The constriction of the traffic light plans, cycles, phase sequences, green light duration and the traffic light synchronization between adjacent intersections are the basis of the traffic control system.

In cities, traffic light control systems are generally of two types: static plan control and adaptive control, that reacts in real time to the traffic variations. The first type of regulation allows the same sequence of phases to be repeated indefinitely with the same duration of the green light, it is less expensive and older, while the second is more efficient and complex. It consists of determining the sequence of green lights according to the actual traffic situation at a single intersection, for an isolated strategy or coordinated strategy in a network of intersections. For normal cases and in the presence of emergency vehicles or accidents.

These adaptive systems respond to the changes in road traffic in order to achieve the following objectives: maximizing traffic flow through intersections during the green light, improving road safety, minimizing vehicles waiting times at intersections, reducing energy consumption and reducing the number of stops that vehicles must make during their journeys on the urban network.

Our solution allows adaptive control of road traffic in a network of adjacent intersections. An intersection among these intersections presented in figure 2 consists mainly of four directions $(\mathrm{N}, \mathrm{S}$, E, W), with three movements for each: go straight, turn left and turn right (figure 1). Turning right will not be considered because vehicles are always allowed to turn right.

Each movement of the intersection is represented by a symbol composed of a combination of two characters. Hence, the two characters represent the cardinal directions ( $\mathrm{N}$ for north, $\mathrm{S}$ for south, $\mathrm{W}$ for west and $\mathrm{E}$ for east) for the source destination. For example, in figure 1, direction $\mathrm{W}$ includes movements: WE, WS and WN.

A traffic phase consists of a set of movements that allows waiting vehicles to cross the intersection at the same time without conflict. With the model used in this work, we have eight possible phases that are presented in figure 3. 
Figure 1. The studied intersection model

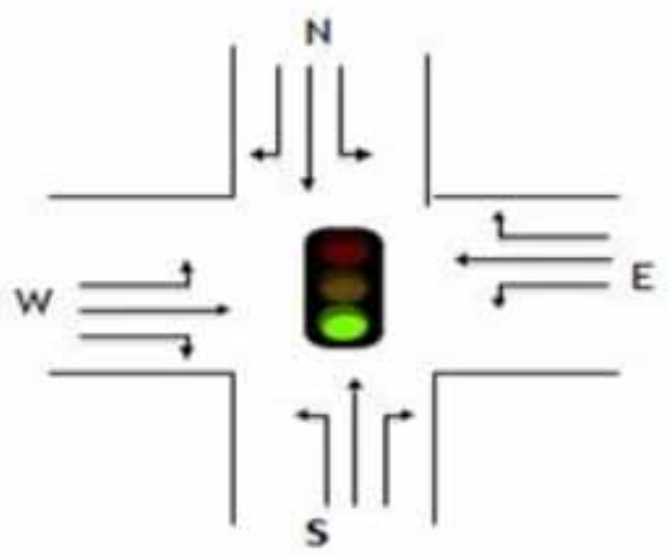

Figure 2. A 6×4 network of traffic intersections

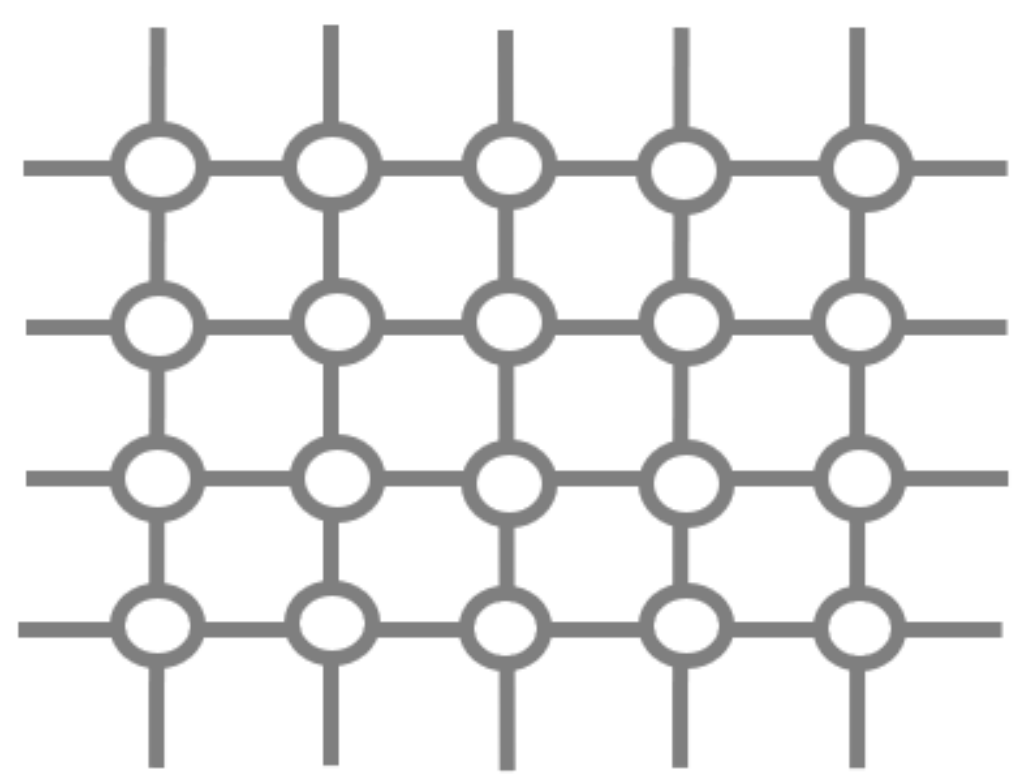

Considering a network of connected intersections, the main idea of our coordinated system is to design the phases of a traffic light cycle in real time, according to the traffic conditions at the intersection concerned and at its neighboring intersections.

The proposed solution consists of three parts, which are illustrated in figure 5: 
Figure 3. The possible phases for the studied intersection

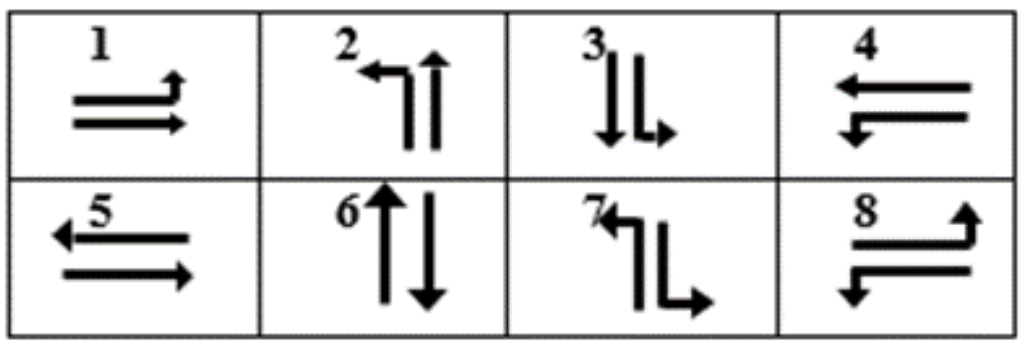

Figure 4. Example of a traffic lights cycle

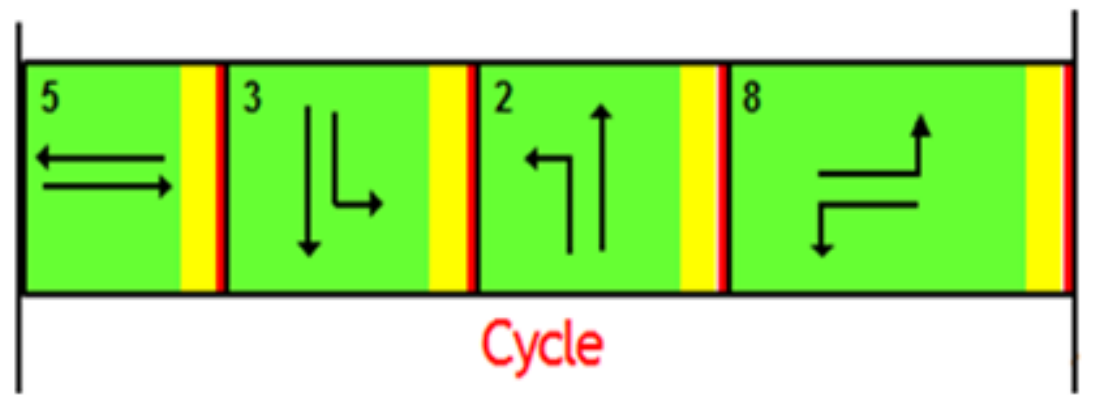

- The first part is devoted to presenting the traffic monitoring system based on a wireless sensor network.

- A second part is meant to determine the sequence of the phases in which we specify the criteria and the process of choice of the next phase.

- And the last one is about calculating the duration of the green light according to the vehicle number of the phase chosen in the second part.

Figure 5. Chart showing the data flow for traffic light controlling

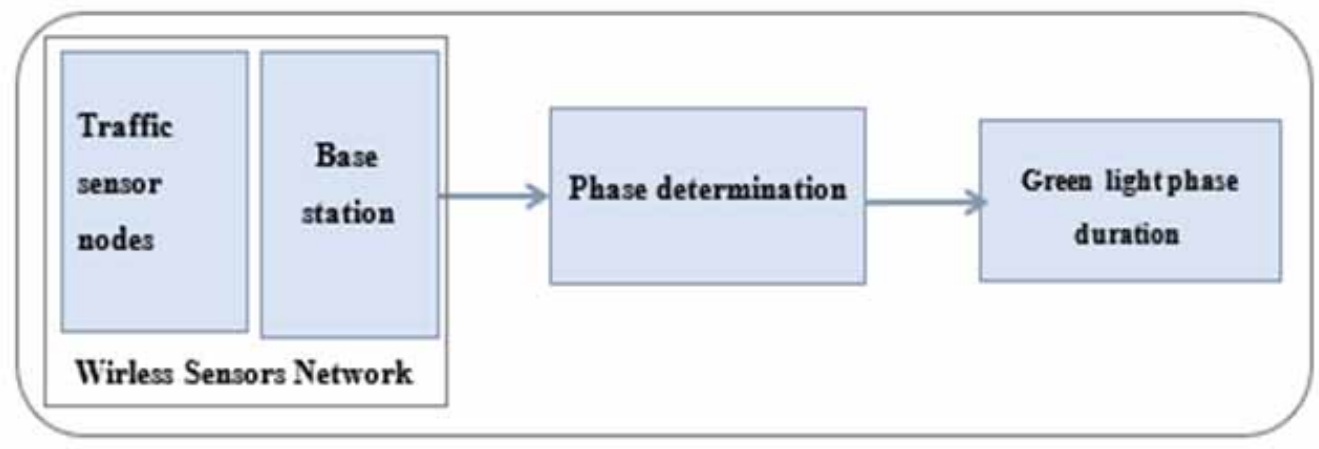


Below, we will explain in details each one of these parts.

\section{NETWORK TOPOLOGY AND DATA COLLECTION}

Traffic knowledge in real time is an essential element for the operation of the road network and in particular to feed the traffic management systems. A wireless sensor network is responsible for collecting traffic data such as: queue lengths and waiting times in each road.

The traffic monitoring system used in this document consists of a number of magnetic sensor nodes installed in the middle of the road. These sensors are used to detect and classify vehicles through evaluating the distortion of the Earth's magnetic field produced by the presence of ferrous objects (Jeong, 2009). It is noteworthy to mention that very few contributions have proposed realistic models for deploying wireless sensor networks in an urban network of intersections as part of an intelligent transport system and the smart city.

Figure 6. WSN topology for an intersection

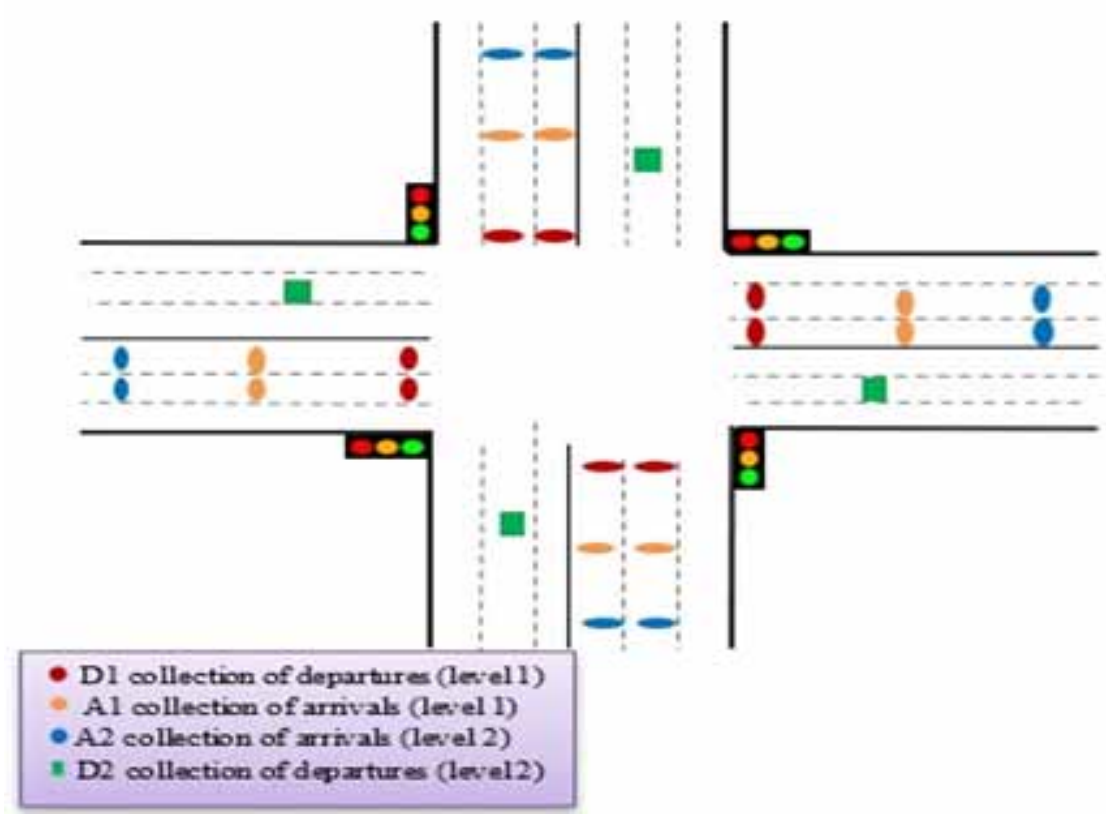

In figure 6, we have shown the proposed topology to control the traffic lights in real time. The topology consists of four nodes per direction, that two of which count the number of arrivals and the others count the number of departures.

In figure 6, we have demonstrated the wireless sensor network topology used to monitor the traffic at an intersection. It consists of four nodes per direction, two of which count the number of arrivals and the others specify the number of departures. Next, we will explain the role of each sensor in our topology and the reason behind choosing a topology with four sensors per direction and not only two sensors as the solutions proposed in (Faye et al., 2012a; Garg \& Kaushal, 2017; Yousef et al., 2010; Zou et al., 2009). 
- Node D1: Allows detecting the number of departures, i.e. the number of vehicles that have left the queue to cross the intersection.

- Nodes A1 and A2: count the number of arrivals. The events detected by these two sensors and those with sensor D1 allow the queue to be calculated and classified the traffic flow into two classes: normal class for a queue located between node D1 and A1, and an important class for a queue that goes from node A1 to node A2.

- Node D2: Specifies the number of vehicles leaving the local interaction to the nearby intersection. The information collected by this sensor will be transmitted to the next intersection on the same lane.

In addition to the other information specified by a topology with two sensors per direction which are waiting time and queue lengths, with a topology of four nodes per lane, it is possible to classify the flow and determine the number of vehicles leaving the intersection to another, so that the latter can predict the number of arrivals.

The distance between sensors on the same input path depends on the number of vehicles that defines the size of these flow classes. For a normal class, the distance between sensors D1 and A1 is calculated according to the number of vehicles defining this class multiplied by the average length of the vehicles, which is 6 meters. For the distance between the A1 and A2 sensors can be defined, for example, as a distance capable of detecting a queue of 25 vehicles. These values can be adjusted according to the specification of the number of vehicles define each flow class.

The events captured by these sensors will be communicated to a decision node via their wireless interfaces. This node is responsible for collecting traffic data from the local intersection and traffic data sent from nearby intersections to produce a traffic signal management decision. It also allows traffic data to be shared with other nearby intersections via wired or wireless communication.

\section{NEXT PHASE DETERMINATION}

In the case of a coordinated system for adjacent intersections, it is necessary to predict the number of arrivals at an intersection at any time using the number of vehicles that have left the adjacent intersections towards that intersection.

Since it is very difficult to instantly assess the exact traffic situation at all intersections due to of its very dynamic environment, we worked with the following assumptions:

- The travel time of a vehicle between two adjacent intersections has a constant value. The travel is expressed according to the following equation:

Travel $=V_{\text {avg }} / d$

- By "Travel" means the travel time, Varg is the average speed of the vehicle between the two intersections that is estimated constant, and $\mathrm{d}$ is the distance between the two intersections.

- Flow conservation, i.e. the number of vehicles entering one roadway front is the same number of vehicles leaving the other roadway front.

Our proposed solution, in this work, consists of designing the traffic light cycle phases according to the traffic conditions at the local intersection concerned by this control, and at the adjacent intersections (figure 7). The wireless sensor network presented in the previous section collects traffic information in different directions, they are in the operation of this algorithm.

In figure 7, we show an example of the interactions between the intersection at the center $\mathrm{Li}$ and its neighbors, and for the movements (WS, WE, WN). The intersection Si represents the source of vehicles that arrives at these movements from the intersection Li. Di1, Di2, and Di3 are the 


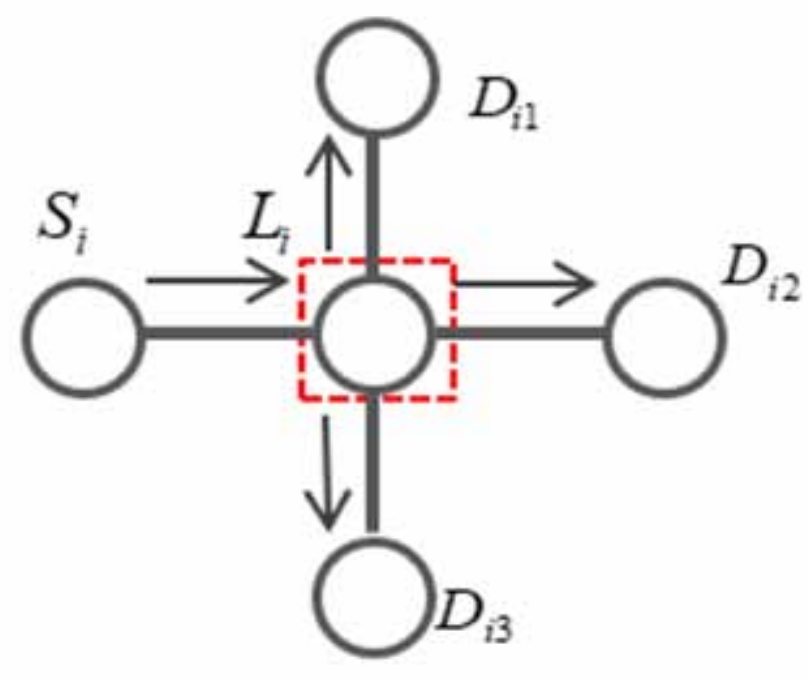

intersections destination of the vehicles coming from Li. Arrows in the figure indicate the traffic flow between these intersections.

In order to introduce the influence of traffic condition at each of these intersections into the controlling decisions at the intersection $\mathrm{Li}$, we have defined three factors for each movement in this intersection.

\section{Factors}

\section{Factor F1:}

This is an indicator of traffic conditions at the local intersection. It allows privileging movements with large queues in the choice of the next phase that will have the green light. This indicator is calculated according to the following two equations.

$$
F_{1}=\frac{Q_{j}}{\sum_{0}^{11} Q_{k}}
$$

$Q_{j}(t)=A R_{j}(t)+A G_{j}(t)+Q_{j}(t-1)-D P_{j}(t)$

$\sum_{0}^{11} Q_{k}$, is the number of vehicles in all the movement of the intersection.

\section{Factor F2:}

At the beginning of each phase, the controller must predict the number of vehicles that will arrive at the intersection before the end of the next phase. The role of this factor is to minimize the 
number of stops at intersections, synchronize traffic lights between intersections and create green waves. To calculate it, it is first necessary to determine the number of vehicles that have already left the intersection $\mathrm{Si}$ to $\mathrm{Li}$, and trying to reach $\mathrm{Li}$ before the end of the next phase that have a green light duration required for the waiting queue calculated by the factor F1.

To specify the value of F2, the controller uses the data captured by sensor D2 at the intersection Si.

Figure 8. The travel of two vehicles v1 and v2 between two intersections as a function of time

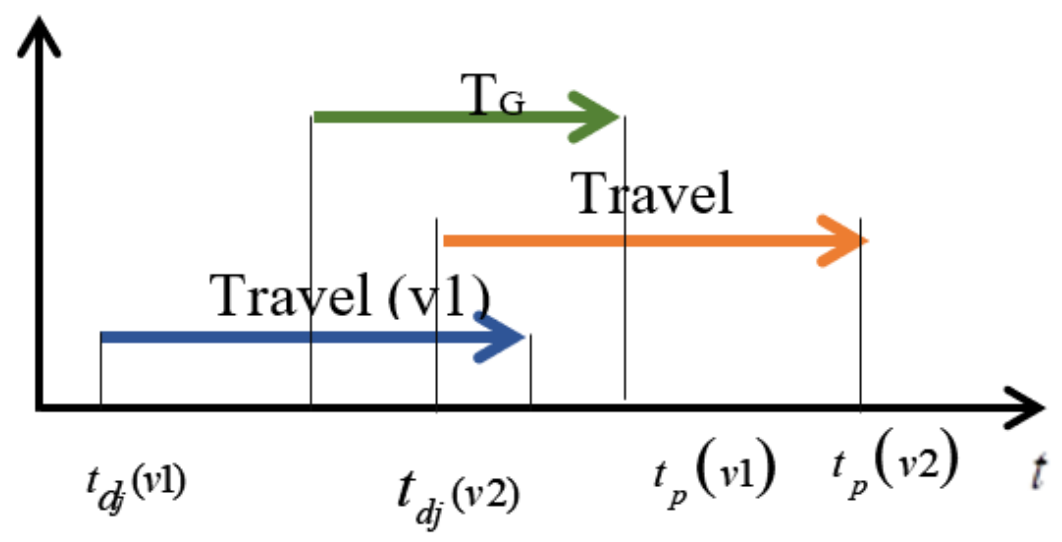

In the following figure 8 , we show the time variations in the movements of the two vehicles v1 and $\mathrm{v} 2$ between the intersections $\mathrm{Si}$ and $\mathrm{Li}$.

Any vehicle verifies the following inequality shall be considered in the factor F2.

$t_{p} \leq t_{d j}+$ Travel $<t_{p}+G T$

With, tp is the starting time of the next phase, and tdk is the moment when the vehicle $\mathrm{k}$ left the last intersection. Travel is the travel time between the two intersections.

The green time for the next phase GT is estimated according to the following equation:

$G T=4+2 * Q_{j}$

Qj is the length of the largest queue of movements of the next phase.

Provided that $\mathrm{Nk}$ is the number of expected arrivals during the phase, the movement $\mathrm{K}$ and $\sum_{0}^{11} N_{k}$ are the numbers planned for the entire intersection. F2 is given as follows: 


$$
F_{2}=\frac{N_{j}}{\sum_{0}^{11} N_{k}}
$$

\section{Factor F3:}

This third factor indicates the congestion degree at destination intersections. It allows the controller to focus on movements leading to slightly loaded intersections rather than congested intersections.

$$
F_{3}=\frac{1}{Q_{j}} \text { if } Q_{j} \neq 0 \text { else } F_{3}=1
$$

$\mathrm{Qj}$ is the number of vehicles at the destination intersection, and precisely in the lane connecting the intersection Li to Di.

After calculating the three factors for each movement, we determine them for all phases of the intersection concerned by this control and which are illustrated in figure 4 .

For a phase $\mathrm{P}$ composed of movements (M1, M2), the three factors (F1, F2, F3) are calculated as follows:

$$
\begin{aligned}
& F_{1}(P)=\frac{F_{1}(M 1)+F_{1}(M 2)}{2} \\
& F_{2}(P)=\frac{F_{2}(M 1)+F_{2}(M 2)}{2} \\
& F_{3}(P)=\frac{F_{3}(M 1)+F_{3}(M 2)}{2}
\end{aligned}
$$

For each phase $\mathrm{P}$, a score $\mathrm{S}(\mathrm{P})$ will be defined as the weighted sum of these three factors, according to the following equation.

$$
S(P)=\frac{\alpha F_{1}(P)+\beta F_{2}(P)+\gamma F_{3}(P)}{\alpha+\beta+\gamma}
$$

$\alpha, \beta$ and $\gamma$ are three weights that can be used to give complementary importance to each of these factors.

\section{Selection Procedure of The Next Phase}

Finally, at the end of each phase, the controller chooses the phase that will be given the green light according to the following rules: 
- Case 1: If traffic flow class at the local intersection is of the important class(F1>Icongestion the degree of congestion), then only the congestion problem at that intersection is addressed. Thus, the next phase will be the phase with the largest F1, i.e. the phase with the largest queue.

- Case 2: If the first case is not achieved, we start at the third factor F3 to determine the degree of congestion at the destination intersections.

- If F3 exceeds a threshold that defines the critical state of congestion(F3>Icritical), then all directions from the local intersection to this congested intersection will be eliminated from the next phase selection and the third case will follow.

- Case 3: If the first case is not realized, then the next phase will be the phase with the maximum score defined in equation 11 .

- Case 4: For an isolated intersection that has no F2 and F3, the decision is based only on the first factor F1, from which the next phase will be the phase with the largest F1.

\section{SIMULATION AND EVALUATION}

In order to evaluate the performance of our approach, accurate information on vehicles travelling at an intersection is necessary. Ideally, our model should have been tested on existing crossroads and compared its performance against the solutions discussed in the literature review. Unfortunately, we do not have access to such intersections, and we do not have enough information on any models to replicate them and compare their results with ours. Therefore, we used the SUMO simulator (Behrisch et al., 2011), which models the behavior of vehicles at the various crossroads flows. This simulator allows us to reproduce user behavior by generating observations that would normally come from the detectors.

Our simulation tool allows determining a set of information about each vehicle, such as: waiting time, position, fuel consumption, $\mathrm{CO} 2$ emission, etc. The behavior of vehicles varies according to

Figure 9. The controlled intersection in SUMO

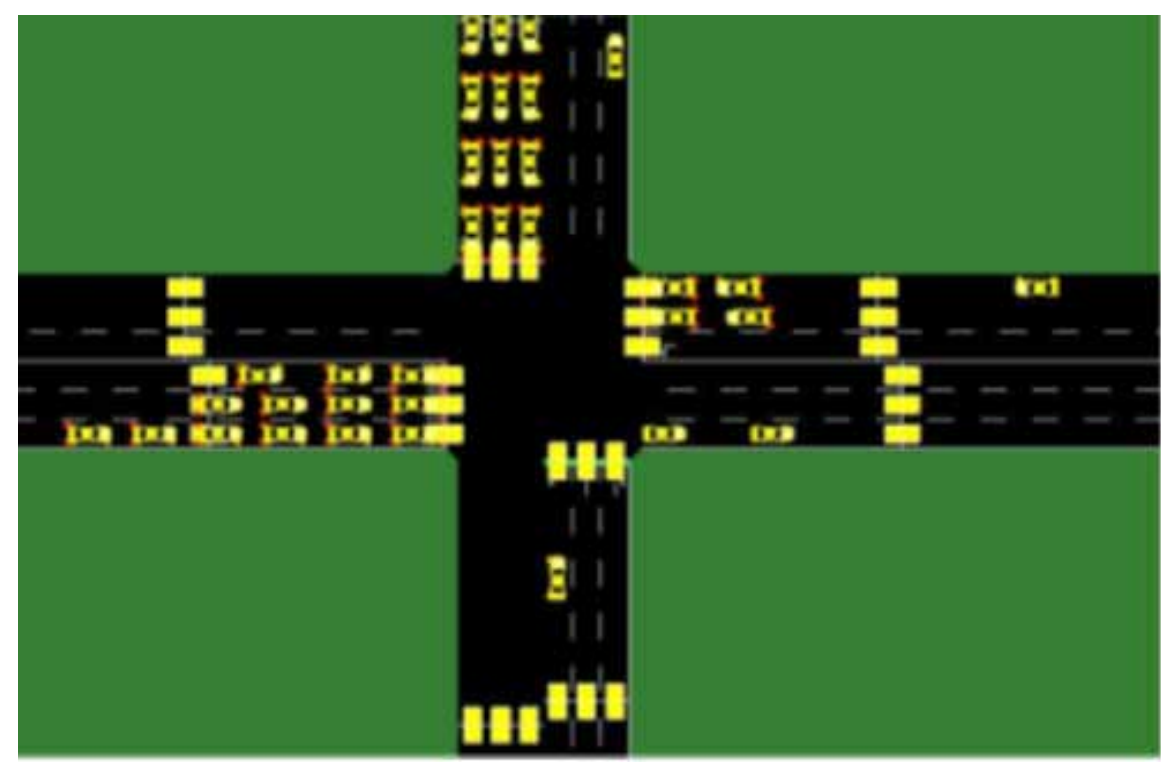


the type of flow to which they belong (turns left, turns right and goes straight), their initial position and the condition of the traffic light.

We tested our approach in a network of intersections, $6 \times 4$ intersections (figure 9), separated by a distance of $1 \mathrm{~km}$.

The simulations are performed for duration of 1300 seconds, and 3600 vehicles enter intersections according to a constant fish distribution between 0.1 and 0.05 .

As in reality, vehicles are considered to enter the coverage area of a crossroads and a network of adjacent crossroads continuously, the simulation of continuous flows must be performed to test the influence of the proposed algorithms on traffic. The four control criteria (average waiting time at an intersection, average queue length, fuel consumption and $\mathrm{CO} 2$ emission) will be studied separately to test the influence of our traffic control approach on these criteria.

Finally, we will also compare the simulation results of our approach with some existing regulation strategy results that are:

- A fixed time controller with four phases of 25- or 20-seconds.

- The TSTMA algorithm proposed in (Yousef et al., 2010), which prioritizes the lanes with the largest queue (isolated strategy).

- The SPF method, which uses the smallest queue (isolated strategy) as the criterion for choosing the next phase.

- Adaptive TOPIOCA algorithm for connecting intersections, it is proposed in (Faye et al., 2012a) (coordinated strategy).

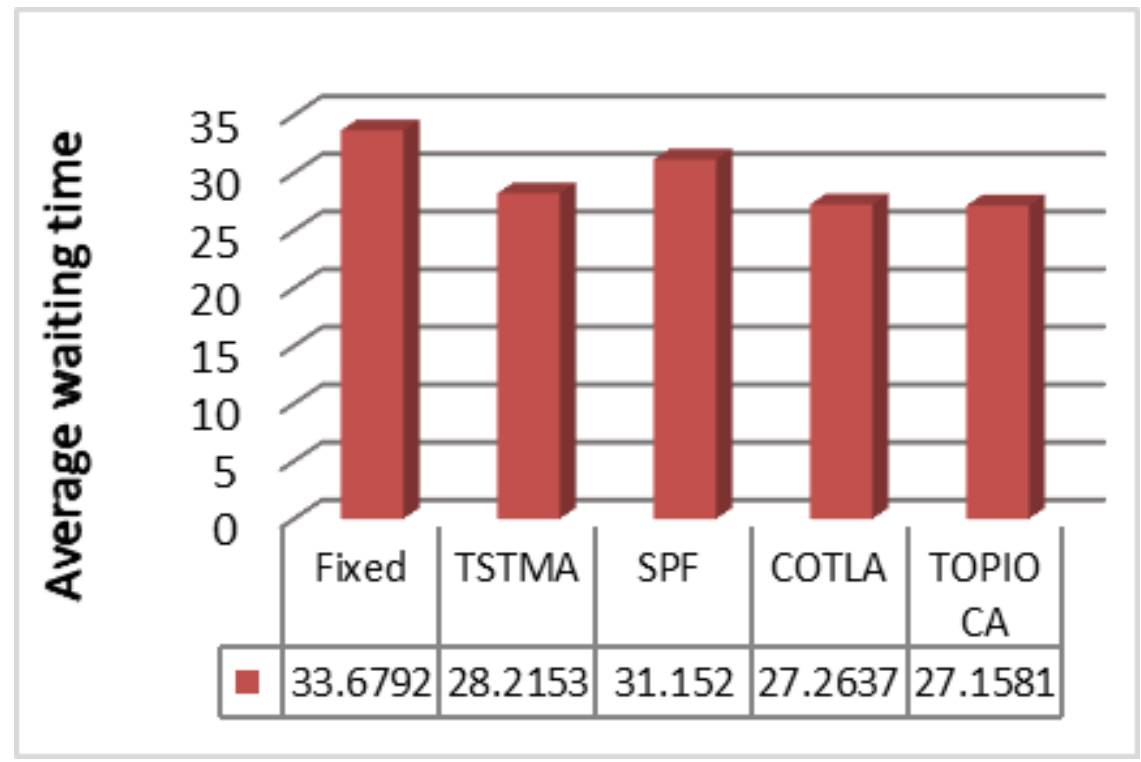

Figure 10 shows the average waiting time for each vehicle in the simulated road network and according to the five methods used. Clearly, our approach is working best. Our algorithm can reduce the average waiting time to $33 \%, 11 \%, 23 \%$ and $7 \%$ respectively for static controller, ASTMA, SPF and TOPIOCA algorithms. 


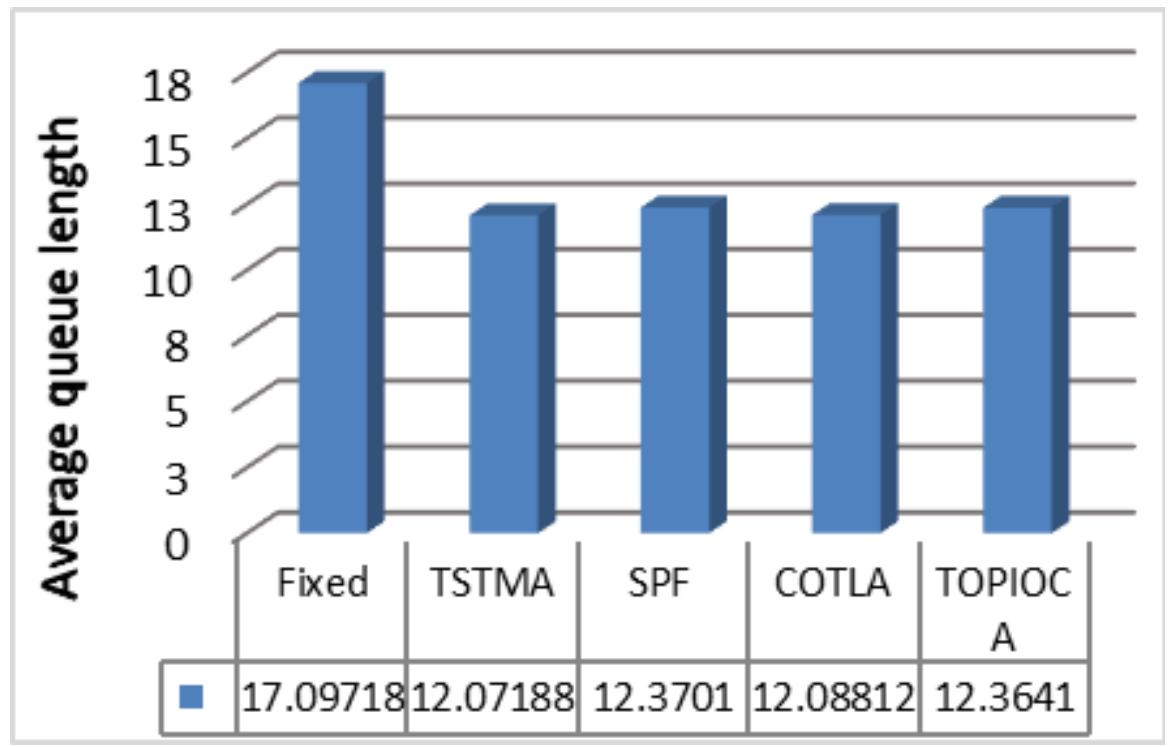

Figure 12. $\mathrm{CO} 2$ emission $(\mathrm{kg})$ with the five approaches

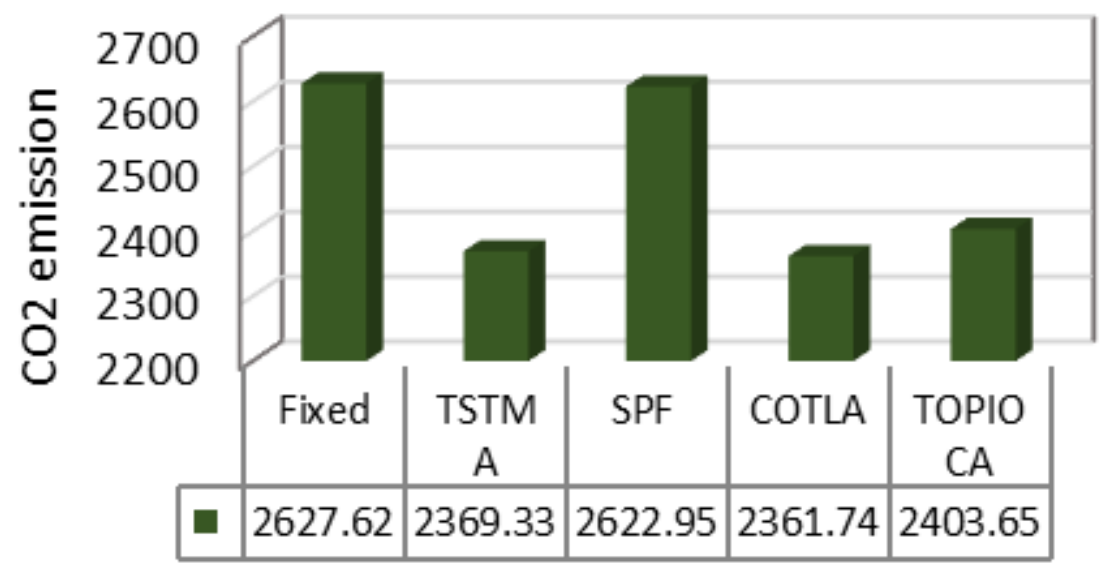

While the algorithm achieves the best results by reducing the average queue length (figure 11). However, the reduction rate is very low compared to the one obtained by our COTLA algorithm, which is $0.1 \%$. Our algorithm has a reduction rate of $41 \%$ in the average queue length compared to the static controller and $2 \%$ compared to SPF and TOPIOCA.

Figure 14 shows the number of vehicles arriving at their destinations during the simulation time and with the five methods. The results show that the largest numbers of arrivals are obtained by our COTLA method followed by the TSTMA and TOPIOCA methods. This means that a road network with traffic controllers using our COTLA algorithm is less loaded than a traffic network with one of the other controllers. 
Figure 13. The fuel consumption in liters

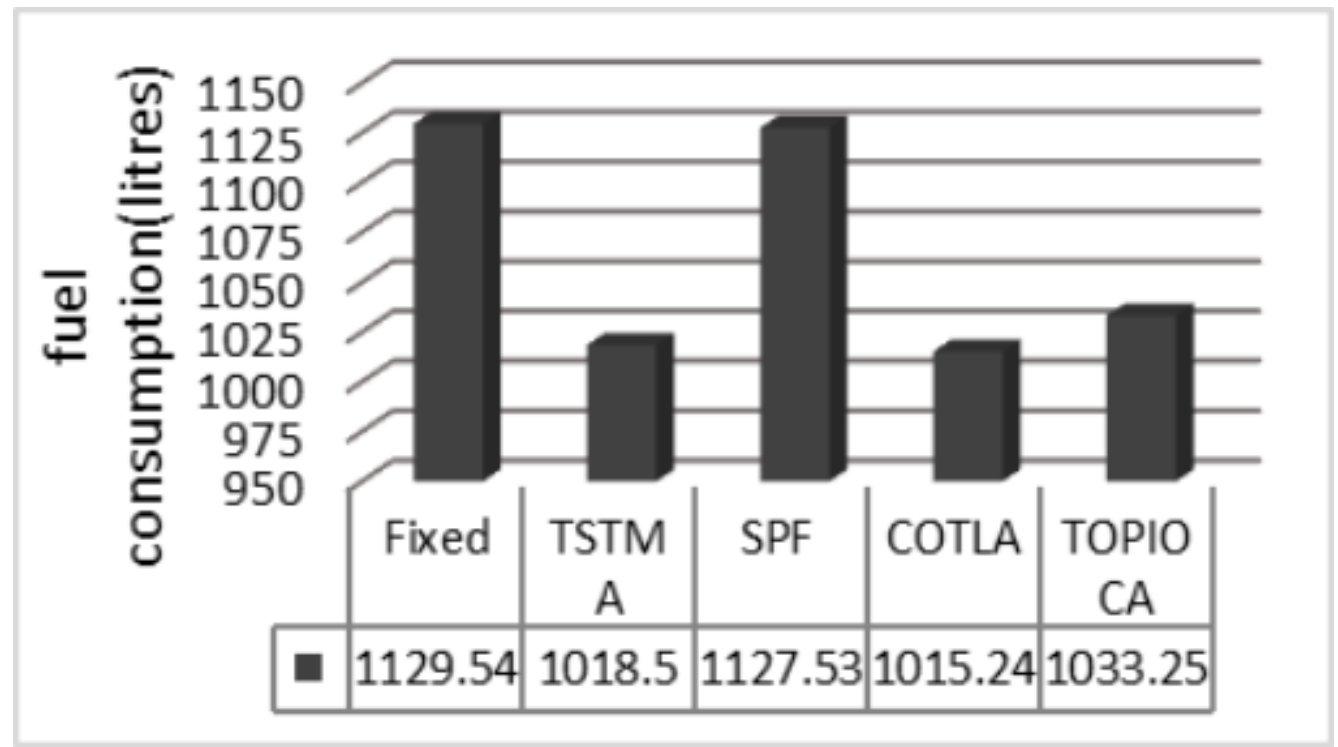

Figure 14. The arrivals numbers with the five approaches

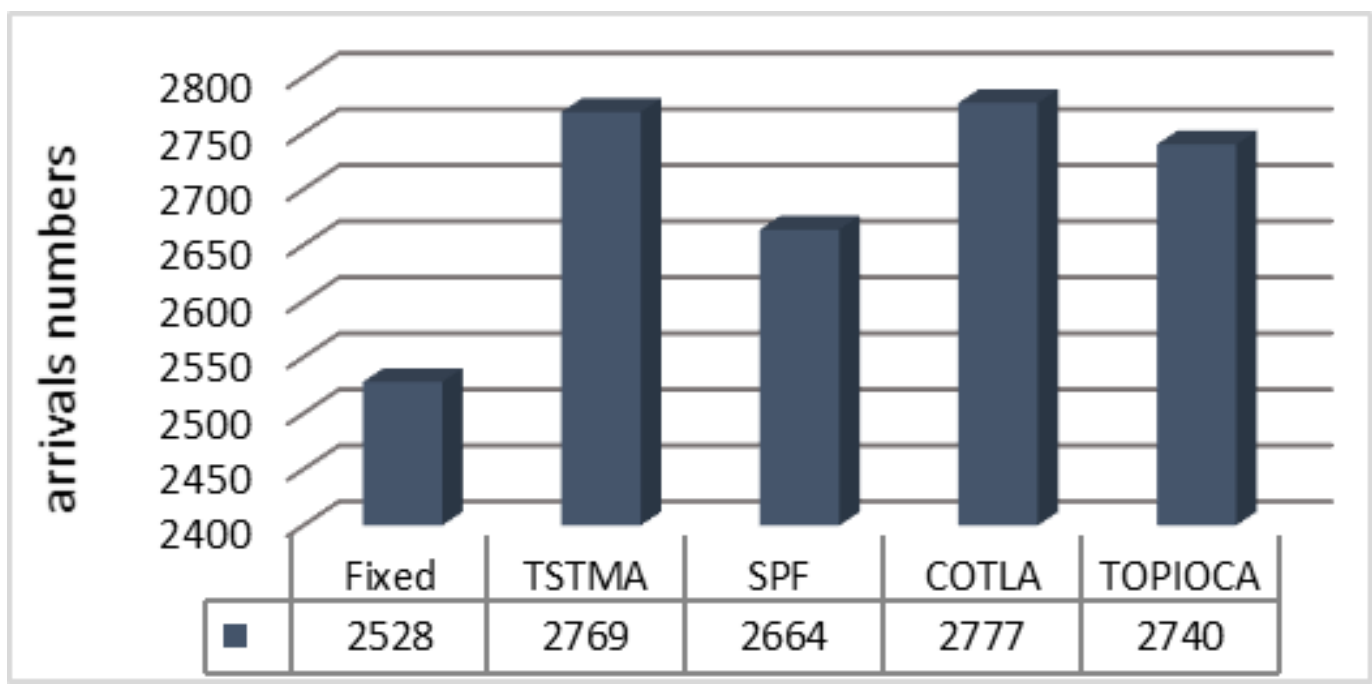

Table 1. The performance of COTLA algorithm compared to other approaches

\begin{tabular}{|l|l|l|l|l|}
\hline & Static & TSTMA & SJF & TOPIOCA \\
\hline The total waiting time & $19 \%$ & $3 \%$ & $12 \%$ & $-0.3 \%$ \\
\hline The queue length & $29 \%$ & $-0.13 \%$ & $2 \%$ & $2 \%$ \\
\hline The arrival numbers & $10 \%$ & $0.28 \%$ & $4.24 \%$ & $1.35 \%$ \\
\hline $\begin{array}{l}\text { The CO2 emission (kg) and } \\
\text { The fuel consumption (liters) }\end{array}$ & $10 \%$ & $0.3 \%$ & $10 \%$ & $1.7 \%$ \\
\hline
\end{tabular}


Figure 12-13 respectively represents the volume of the fuel consumption and the amount emitted in $\mathrm{CO} 2$ by the vehicles in the simulation. The results show that COTA and TSTMA give the lowest fuel consumption and $\mathrm{CO} 2$ emission with the same reduced rates for both: $10 \%$ compared to the fixed-time controls, $17 \%$ for the TOPIOCCA algorithm and 1\% compared to the SPF.

The discussed performances of our solution are presented in the table below:

In summary, our coordinated controller for a connected intersection network reduces: the network load, the waiting time, the energy consumption and the $\mathrm{CO} 2$ emissions better than adaptive controllers in isolated intersections or fixed-time controllers. Indeed, the weakness of isolated strategies is that they control traffic according to the variations in traffic in the intersection concerned; they do not try to predict arrivals at intersections. However, our solution manages traffic according to the state of traffic in the concerned intersection and its neighbors in order to meet the overall objectives of controlling a geographical area.

\section{CONCLUSION}

In this article, we have proposed a coordinated traffic light control approach based wireless sensor network. The proposed solution cooperates and adapt the control policy to the traffic conditions at multiple intersections. in addition to adapt the traffic lights to the traffic conditions in the concerned intersection, the algorithm predicts new arrivals and calculates the degree of congestion in neighboring intersections, in order to encourage sending flows of vehicles to intersections that are less dense.

The simulations results demonstrate the efficiency and practicality of our approach to reduce average vehicle waiting times and queue lengths. And the obtained performances show that the proposed algorithm is better than the fixed time control and the isolated strategies.

In the future work, we will build networks based on several intersection models with different traffic scenarios in order to evaluate our solution under different conditions. In addition, we will equip the traffic monitoring system with the ability to detect the presence of priority vehicles, such as the presence of the police, public transport, trucks, ambulance, etc. 


\section{REFERENCES}

Ahmad, F., Mahmud, S. A., \& Yousaf, F. Z. (2016). Shortest processing time scheduling to reduce traffic congestion in dense urban areas. IEEE Transactions on Systems, Man, and Cybernetics. Systems, 47(5), 838-855. doi:10.1109/TSMC.2016.2521838

Aslani, M., Seipel, S., \& Wiering, M. (2018). Continuous residual reinforcement learning for traffic signal control optimization. Canadian Journal of Civil Engineering, 45(8), 690-702. doi:10.1139/cjce-2017-0408

Barzilay, O., Voloch, N., Hasgall, A., Lavi Steiner, O., \& Ahituv, N. (2018). Traffic Control in a Smart Intersection by an Algorithm with Social Priorities. Contemporary Engineering Sciences, 11(31), 2018. doi:10.12988/ ces. 2018.83126

Behrisch, M., Bieker, L., Erdmann, J., \& Krajzewicz, D. (2011). SUMO-simulation of urban mobility: an overview. In Proceedings of SIMUL 2011, The Third International Conference on Advances in System Simulation. ThinkMind.

Belbachir, A., El Fallah-Seghrouchni, A., Casals, A., \& Pasin, M. (2019). Smart mobility using multi-agent system. Procedia Computer Science, 151, 447-454. doi:10.1016/j.procs.2019.04.061

Belkeziz, R., \& Jarir, Z. (2020). An Overview of the IoT Coordination Challenge. International Journal of Service Science, Management, Engineering, and Technology, 11(1), 99-115. doi:10.4018/IJSSMET.2020010107

Burguillo-Rial, J. C., Rodriguez-Hernandez, P. S., Montenegro, E. C., \& Castiñeira, F. G. (2012). History-based self-organizing traffic lights. Computer Information, 28(2), 157-168.

Collotta, M., Bello, L. L., \& Pau, G. (2015). A novel approach for dynamic traffic lights management based on Wireless Sensor Networks and multiple fuzzy logic controllers. Expert Systems with Applications, 42(13), 5403-5415. doi:10.1016/j.eswa.2015.02.011

Faye, S., Chaudet, C., \& Demeure, I. (2012a, December). A distributed algorithm for multiple intersections adaptive traffic lights control using a wireless sensor networks. In Proceedings of the first workshop on Urban networking (pp. 13-18). doi:10.1145/2413236.2413240

Faye, S., Chaudet, C., \& Demeure, I. (2012b, September). A distributed algorithm for adaptive traffic lights control. In 2012 15th International IEEE Conference on Intelligent Transportation Systems (pp. 1572-1577). IEEE. doi:10.1109/ITSC.2012.6338671

Fayez, R., Taha, M. T. A. E., \& Gadallah, M. (2020). Occluded Object Tracking System (OOTS). International Journal of Service Science, Management, Engineering, and Technology, 11(3), 65-81. doi:10.4018/ IJSSMET.2020070105

Gao, K., Zhang, Y., Sadollah, A., \& Su, R. (2016). Optimizing urban traffic light scheduling problem using harmony search with ensemble of local search. Applied Soft Computing, 48, 359-372. doi:10.1016/j. asoc.2016.07.029

Garg, H., \& Kaushal, E. G. (2017). Traffic Lights Control System for Indian Cities Using WSN and Fuzzy Control. Traffic (Copenhagen, Denmark), 4(07), 56-65.

Ge, H., Song, Y., Wu, C., Ren, J., \& Tan, G. (2019). Cooperative deep Q-learning with Q-value transfer for multiintersection signal control. IEEE Access: Practical Innovations, Open Solutions, 7, 40797-40809. doi:10.1109/ ACCESS.2019.2907618

Ha-li, P., \& Ke, D. (2017, October). An intersection signal control method based on deep reinforcement learning. In 2017 10th International Conference on Intelligent Computation Technology and Automation (ICICTA) (pp. 344-348). IEEE. doi:10.1109/ICICTA.2017.83

Helbing, D., Lämmer, S., \& Lebacque, J. P. (2005). Self-organized control of irregular or perturbed network traffic. In Optimal control and dynamic games (pp. 239-274). Springer. doi:10.1007/0-387-25805-1_15

Jeong, J. (2009). Wireless sensor networking for intelligent transportation systems. Academic Press.

Jin, J., \& Ma, X. (2018). Hierarchical multi-agent control of traffic lights based on collective learning. Engineering Applications of Artificial Intelligence, 68, 236-248. doi:10.1016/j.engappai.2017.10.013 
Jin, J., \& Ma, X. (2019). A multi-objective agent-based control approach with application in intelligent traffic signal system. IEEE Transactions on Intelligent Transportation Systems, 20(10), 3900-3912. doi:10.1109/ TITS.2019.2906260

Jing, P., Huang, H., \& Chen, L. (2017). An adaptive traffic signal control in a connected vehicle environment: A systematic review. Information (Basel), 8(3), 101. doi:10.3390/info8030101

Kell, J. H., \& Fullerton, I. J. (1991). Manual of traffic signal design. Academic Press.

Khanafer, M., Guennoun, M., \& Mouftah, H. T. (2009, December). WSN architectures for intelligent transportation systems. In 2009 3rd International Conference on New Technologies, Mobility and Security (pp. 1-8). IEEE. doi:10.1109/NTMS.2009.5384685

Kumaran, S. K., Mohapatra, S., Dogra, D. P., Roy, P. P., \& Kim, B. G. (2019). Computer vision-guided intelligent traffic signaling for isolated intersections. Expert Systems with Applications, 134, 267-278. doi:10.1016/j. eswa.2019.05.049

Li, L., Lv, Y., \& Wang, F. Y. (2016). Traffic signal timing via deep reinforcement learning. IEEE/CAA Journal of Automatica Sinica, 3(3), 247-254.

Li, Z., Shahidehpour, M., Bahramirad, S., \& Khodaei, A. (2016). Optimizing traffic signal settings in smart cities. IEEE Transactions on Smart Grid, 8(5), 2382-2393. doi:10.1109/TSG.2016.2526032

Liang, X., Du, X., Wang, G., \& Han, Z. (2019). A deep reinforcement learning network for traffic light cycle control. IEEE Transactions on Vehicular Technology, 68(2), 1243-1253. doi:10.1109/TVT.2018.2890726

Liu, Y., Liu, L., \& Chen, W. P. (2017, October). Intelligent traffic light control using distributed multi-agent Q learning. In 2017 IEEE 20th International Conference on Intelligent Transportation Systems (ITSC) (pp. 1-8). IEEE. doi:10.1109/ITSC.2017.8317730

Mousavi, S. S., Schukat, M., \& Howley, E. (2017). Traffic light control using deep policy-gradient and valuefunction-based reinforcement learning. IET Intelligent Transport Systems, 11(7), 417-423. doi:10.1049/ietits. 2017.0153

Odeh, S. M. (2013). Management of an intelligent traffic light system by using genetic algorithm. Journal of Image and Graphics, 1(2), 90-93. doi:10.12720/joig.1.2.90-93

Rida, N., \& Hasbi, A. (2018a, October). Traffic Lights Control using Wireless Sensors Networks. In Proceedings of the 3rd International Conference on Smart City Applications (pp. 1-6). doi:10.1145/3286606.3286791

Rida, N., \& Hasbi, A. (2018b, October). Dynamic Traffic Lights Control for Isolated Intersection Based Wireless Sensor Network. In The Proceedings of the Third International Conference on Smart City Applications (pp. 1036-1044). Springer.

Rida, N., Ouadoud, M., \& Hasbi, A. (2020a). Ant colony optimization for real time traffic lights control on a single intersection. Academic Press.

Rida, N., Ouadoud, M., \& Hasbi, A. (2020b). Traffic Signal Control for a Single Intersection-Based Intelligent Transportation System. In Digital Transformation and Innovative Services for Business and Learning (pp. 159-180). IGI Global. doi:10.4018/978-1-7998-5175-2.ch009

Rida, N., Ouadoud, M., Hasbi, A., \& Chebli, S. (2018, October). Adaptive traffic light control system using wireless sensors networks. In 2018 IEEE 5th International Congress on Information Science and Technology (CiSt) (pp. 552-556). IEEE. doi:10.1109/CIST.2018.8596620

Rithesh, R. N., Vignesh, R., \& Anala, M. R. (2018). Autonomous Traffic Signal Control using Decision Tree. International Journal of Electrical \& Computer Engineering, 8(3).

Salman, M. A., Ozdemir, S., \& Celebi, F. V. (2018). Fuzzy traffic control with vehicle-to-everything communication. Sensors (Basel), 18(2), 368. doi:10.3390/s18020368 PMID:29382053

Shi, M. K., Jiang, H., \& Li, S. H. (2016, November). An intelligent traffic-flow-based real-time vehicles scheduling algorithm at intersection. In 2016 14th International Conference on Control, Automation, Robotics and Vision (ICARCV) (pp. 1-5). IEEE. doi:10.1109/ICARCV.2016.7838779 
Spall, J. C., \& Chin, D. C. (1997). Traffic-responsive signal timing for system-wide traffic control. Transportation Research Part C, Emerging Technologies, 5(3-4), 153-163. doi:10.1016/S0968-090X(97)00012-0

Tan, M. K., Chuo, H. S. E., Chin, R. K. Y., Yeo, K. B., \& Teo, K. T. K. (2018, November). Hierarchical Multiagent System in Traffic Network Signalization with Improved Genetic Algorithm. In 2018 IEEE International Conference on Artificial Intelligence in Engineering and Technology (IICAIET) (pp. 1-6). IEEE. doi:10.1109/ IICAIET.2018.8638464

Tubaishat, M., Qi, Q., Shang, Y., \& Shi, H. (2008, January). Wireless sensor-based traffic light control. In 2008 5th IEEE Consumer Communications and Networking Conference (pp. 702-706). IEEE. doi:10.1109/ccnc08.2007.161

Van der Pol, E., \& Oliehoek, F. A. (2016). Coordinated deep reinforcement learners for traffic light control. Proceedings of Learning, Inference and Control of Multi-Agent Systems (at NIPS 2016).

Wei, H., Zheng, G., Yao, H., \& Li, Z. (2018, July). Intellilight: A reinforcement learning approach for intelligent traffic light control. In Proceedings of the 24th ACM SIGKDD International Conference on Knowledge Discovery \& Data Mining (pp. 2496-2505). doi:10.1145/3219819.3220096

Yang, Q., \& Koutsopoulos, H. N. (1996). A microscopic traffic simulator for evaluation of dynamic traffic management systems. Transportation Research Part C, Emerging Technologies, 4(3), 113-129. doi:10.1016/ S0968-090X(96)00006-X

Yen, C. C., Ghosal, D., Zhang, M., Chuah, C. N., \& Chen, H. (2018, December). Falsified data attack on backpressure-based traffic signal control algorithms. In 2018 IEEE Vehicular Networking Conference (VNC) (pp. 1-8). IEEE. doi:10.1109/VNC.2018.8628334

Younes, M. B., \& Boukerche, A. (2018). An efficient dynamic traffic light scheduling algorithm considering emergency vehicles for intelligent transportation systems. Wireless Networks, 24(7), 2451-2463. doi:10.1007/ s11276-017-1482-5

Younes, M. B., Boukerche, A., \& Mammeri, A. (2016, April). Context-aware traffic light self-scheduling algorithm for intelligent transportation systems. In 2016 IEEE Wireless Communications and Networking Conference. IEEE.

Yousef, K. M., Al-Karaki, M. N., \& Shatnawi, A. M. (2010). Intelligent traffic light flow control system using wireless sensors networks. Journal of Information Science and Engineering, 26(3), 753-768.

Yousef, K. M. A., Shatnawi, A., \& Latayfeh, M. (2019). Intelligent traffic light scheduling technique using calendar-based history information. Future Generation Computer Systems, 91, 124-135. doi:10.1016/j. future.2018.08.037

Zhou, B., Cao, J., Zeng, X., \& Wu, H. (2010, September). Adaptive traffic light control in wireless sensor network-based intelligent transportation system. In 2010 IEEE 72nd Vehicular technology conference-fall. IEEE.

Zhou, P., Braud, T., Alhilal, A., Hui, P., \& Kangasharju, J. (2019, March). ERL: Edge based Reinforcement Learning for optimized urban Traffic light control. In 2019 IEEE International Conference on Pervasive Computing and Communications Workshops (PerCom Workshops) (pp. 849-854). IEEE. doi:10.1109/ PERCOMW.2019.8730706

Zhu, Q., Peng, C., Shi, J., Duan, P., Bao, Y., \& Xie, M. (2016). Cooperative traffic light control based on semireal-time processing. Journal of Automation and Control Engineering, 4(1).

Zhu, Y., Liu, X., Li, M., \& Zhang, Q. (2012). POVA: Traffic light sensing with probe vehicles. IEEE Transactions on Parallel and Distributed Systems, 24(7), 1390-1400. doi:10.1109/TPDS.2012.233

Zou, F., Yang, B., \& Cao, Y. (2009, August). Traffic light control for a single intersection based on wireless sensor network. $20099 t$.

Abderrahim Hasbi got his PhD degree in computer science from the University Mohamed 5 of Rabat Morocco. He's full Professor in Computer Science at the Mohammadia School of Engineering of the University Mohamed 5 Agdal, Morocco. He's member of the Network and Intelligent Systems Group and he has a lot of contributions. 